\title{
AMPLIFICADOR CLASSE D PARA SUBWOOFER
}

\author{
Fabio V. R. da Silva* \\ fabiovince@gmail.com
}

João Batista Vieira Júnior*

batista@ufu.br

\author{
Leandro dos S. Martins* \\ ldsmartins@gmail.com
}

Ernane A. A. Coelho*

ernanedufu.br

\section{Luiz Carlos de Freitas* \\ freitas@ufu.br}

*Universidade Federal de Uberlândia

Faculdade de Engenharia Elétrica - FEELT

Núcleo de Eletrônica de Potência - NuEP

Av. João Naves de Ávila, 2121 - Campus Santa Mônica, Bloco "3N", CEP 38400-902

Uberlândia, MG - Brasil, Phone: +55-34-32394166

\begin{abstract}
Class D Amplifier for Subwoofer

This paper proposal presents a high power Class-D audio amplifier for subwoofer applications with efficiency above $90 \%$. A regulated dc voltage supply is not needed as well as a low pass output filter overcoming the disadvantages of ordinary Class-D audio amplifiers structures. A $1 \mathrm{~kW}$ laboratory prototype was implemented and it was found that efficiency above $90 \%$ and THD below $1 \%$ could be achieved. Detailed design guide lines, principle of operation analysis and control strategy are also included in this paper proposal.
\end{abstract}

KEYWORDS: High efficiency, Class D amplifier, low THD.

\section{RESUMO}

Este artigo apresenta uma topologia de amplificador Classe D de potência adequado para operar com sub-woofer. A estrutura proposta não necessita de fonte de alimentação estabilizada e filtro passa baixas de saída como ocorre com os amplificadores Classe D tradicionais. Um protótipo de $1 \mathrm{~kW}$ foi implementado em laboratório obtendo-se rendimento su-

Artigo submetido em 16/02/2009 (Id.: 00956)

Revisado em 24/04/2009, 18/06/2009, 18/07/2009

Aceito sob recomendação do Editor Associado Prof. Enes Gonçalves Marra perior a $90 \%$ e THD inferior a $1 \%$. Roteiro de projeto, princípio de operação e estratégia de controle também são apresentados.

PALAVRAS-CHAVE: Alto rendimento, amplificador Classe D, baixo THD.

\section{INTRODUCÃO}

Os amplificadores lineares de áudio apresentam vantagens como: baixa distorção e ruído reduzido. No entanto, possuem a desvantagem do peso elevado, baixo rendimento e a necessidade de circuitos complexos com duas ou mais etapas excitadoras em cascata, aumentando o custo do equipamento, a quantidade de distorção e a possibilidade de instabilidade devido à complexidade da função de transferência, Janssen (2004).

Por outro lado, os amplificadores chaveados e os híbridos apresentam rendimento elevado e, dependendo do controle implementado, oferecem baixa distrorção. As principais características destes amplificadores são:

1. Classe $\mathrm{D}$ - os dispositivos de potência destes amplificadores operam no corte ou na saturação. O rendimento máximo teórico do amplificador Classe D é $100 \%$ e ren- 


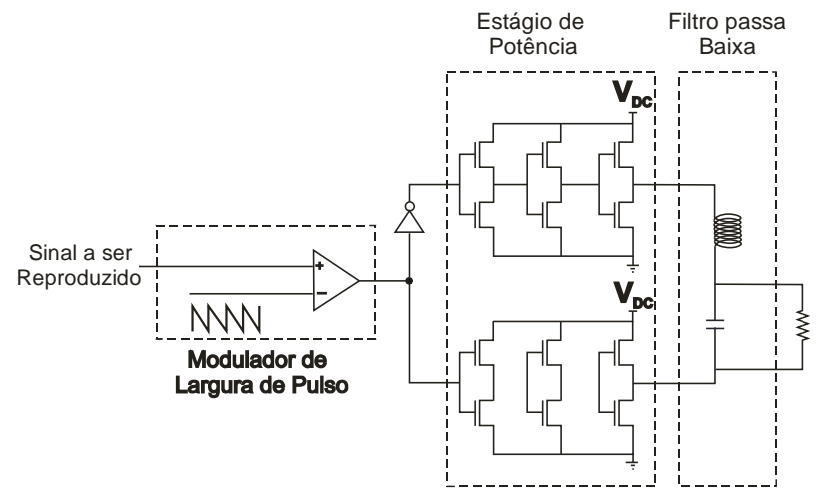

Figura 1: Conversor Classe D convencional.

dimentos em torno de $90 \%$ são obtidos na prática, Mioni (2007). Neste contexto, os amplificadores Classe D possuem rendimento superior aos amplificadores (Classe A, B e AB), Walker (2003).

2. Amplificadores Híbridos (Switch-Mode assisted linear amplifier - SMALA), são amplificadores compostos por um amplificador Classe D e um linear (Classe A, B ou AB), Ertl et alii (2003) e Zee e Tuijl (2003). A idéia de se cascatear um amplificador linear com um chaveado foi apresentada pela primeira vez por Yundt (1986).

Os amplificadores chaveados tradicionais, Fig. 1, são formados por um estágio modulador de largura de pulso (PWM), por um estágio de potência e um filtro passa baixas de saída. No estágio PWM, o sinal de referência é comparado com um sinal dente de serra de alta frequência de modo a se obter os pulsos proporcionais ao sinal de referência amplificado.

O estágio de potência é frequentemente composto por uma estrutura do tipo inversor de tensão em ponte completa de modo que níveis de potência de saída mais elevados possam ser alcançados, principalmente, em aplicações de baixa tensão.

O filtro de saída é usado para remover as componentes harmônicas do sinal PWM de modo a recompor o sinal de referência amplificado que é aplicado na carga.

Buscando-se reduzir o nível de ruído, devido a não linearidade do amplificador chaveado e evitar a distorção causada pelo cruzamento por zero (crossover distortion), inúmeros circuitos, com diferentes topologias e estratégias de controle foram propostos na literatura técnica e científica, Antunes et alii (2007), Gianart et alii (2003), Lai e Smedley (1996), Oliva et alii (2004) e Pascual et alii (2003).

Vale salientar que o sinal PWM gerado depende do nível da fonte de alimentação do circuito de potência. Portanto,

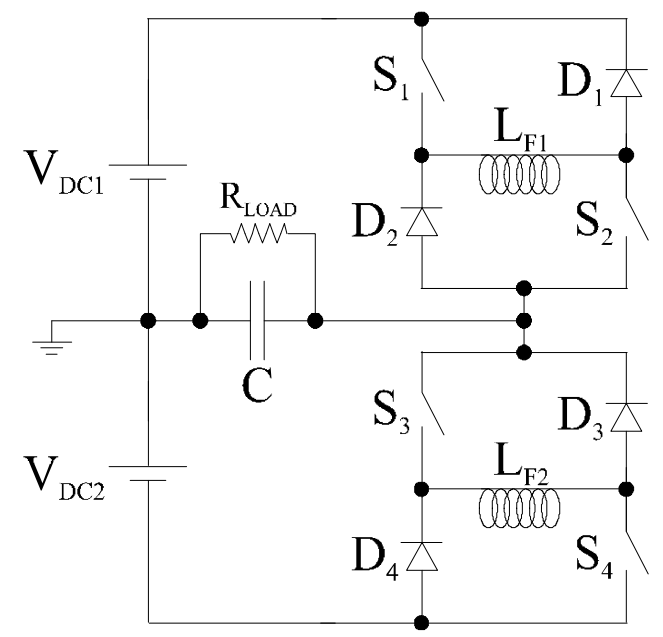

Figura 2: Conversor Classe D apresentado em trabalhos anteriores.

qualquer oscilação no nível da fonte de alimentação afetará o sinal PWM amplificado distorcendo o sinal de áudio. Para solucionar esse problema, um circuito de realimentação deve ser implementado, como mencionado em Chang et alii (2007) e Nilsen (1997).

Em trabalhos anteriores, a topologia exibida na Fig. 2, proposta por Silva et alii (1999a) e Silva et alii (1999b), apresentou uma inovação expressiva, com relação aos amplificadores Classe $\mathrm{D}$ tradicionais, eliminando o filtro passa baixas na saída. Além disso, o controle realimentado foi implementado com um circuito de baixo custo e em caso do disparo simultâneo dos quatro interruptores, devido a ruído, os indutores em série protegem os interruptores. Por outro lado, este arranjo necessita de quatro interruptores e utiliza snubber dissipativo em todos os interruptores.

Com o objetivo de reduzir o número de interruptores da topologia apresentada na Fig. 2 e evitar o uso de snubbers dissipativos, uma nova topologia de amplificador Classe D foi desenvolvida e é apresentada neste trabalho.

As principais vantagens da topologia proposta, mostrada na Fig. 3, com relação a estruturas apresentadas em trabalhos anteriores são:

- A nova topologia utiliza apenas dois interruptores e, consequentemente, dois circuitos de comando de interruptor proporcionando redução de custo;

- Comutação suave é naturalmente obtida, o que reduz a emissão de EMI;

- Snubbers dissipativos são desnecessários. 


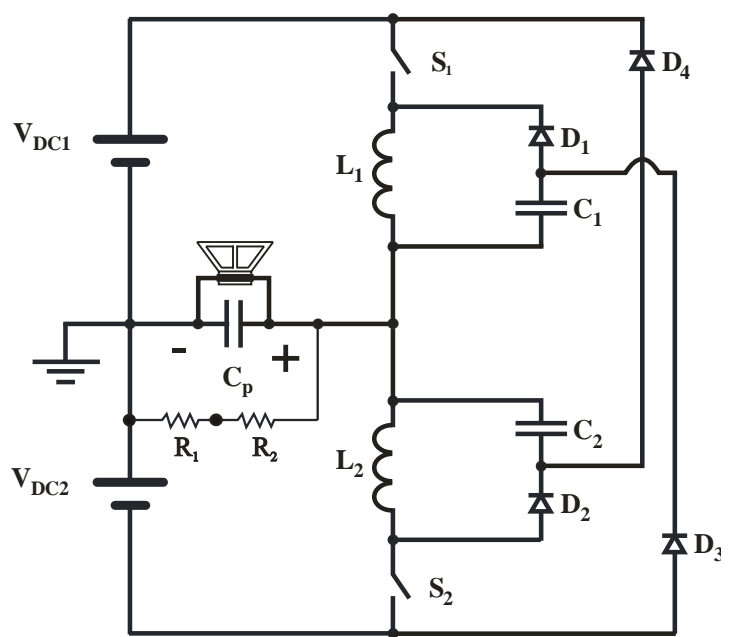

Figura 3: Conversor Classe D proposto.

\section{PRINCÍPIO DE OPERAÇÃO E ESTRA- TÉGIA DE CONTROLE}

Os interruptores $S_{1}$ e $S_{2}$ operam de modo complementar, isto é, quanto o interruptor $S_{1}$ está conduzindo o interruptor $S_{2}$ está aberto e vice-versa.

De modo a introduzir o princípio de operação do amplificador de potência proposto, os estágios de operação serão descritos a seguir. Primeiro, três regras devem ser consideradas:

i O capacitor $C_{1}$ equivale a uma fonte de tensão com a polaridade indicada na Figura $4\left(V_{D C 1}=V_{C P}+V_{D C 2}\right)$;

ii $\mathrm{O}$ capacitor $C_{2}$ equivale a uma fonte de tensão com a polaridade indicada na Figura $4\left(V_{D C 2}=V_{C P}+V_{D C 1}\right)$;

iii Em qualquer instante de tempo a soma das tensões sobre os capacitores $C_{1}$ e $C_{2}$ é igual a soma das tensões $V_{D C 1}$ e $V_{D C 2}\left(V_{C 1}+V_{C 2}=V_{D C 1}+V_{D C 2}\right)$.

Primeira Etapa: durante o semi-ciclo positivo da tensão $V_{C P}$, cuja polaridade está indicada na Fig. 4 (a), a carga recebe energia da fonte de tensão $V_{D C 1}$ através do indutor $L_{1}$. Ao mesmo tempo, o capacitor $\mathrm{C}_{2}$ recebe a energia armazenada em $L_{2}$ e parte desta energia é desviada através de $L_{1}$.

Segunda Etapa: existe um tempo morto durante a abertura de um interruptor e o fechamento de outro, como indicado na Fig. 4 (b). Portanto, o circuito composto por $C_{1}, C_{2}$, $D_{1}$ e $D_{2}$ recebe a energia armazenada nos indutores $L_{1}$ e $L_{2}$ quando os interruptores $S_{1}$ e $S_{2}$ estão simultâneamente abertos. Neste instante, parte da energia é devolvida para as fontes de tensão $V_{D C 1}$ e $V_{D C 2}$ através dos diodos $D_{3}$ e $D_{4}$.

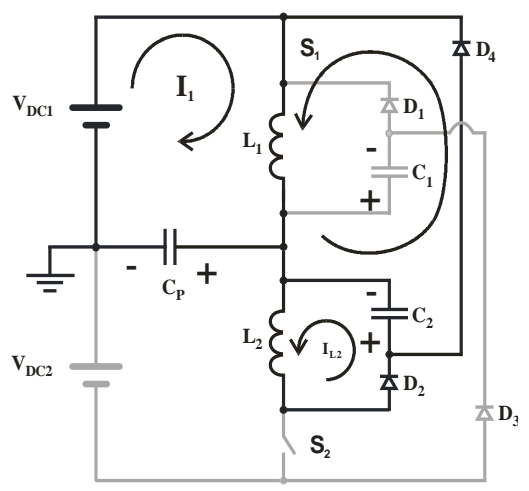

( a )

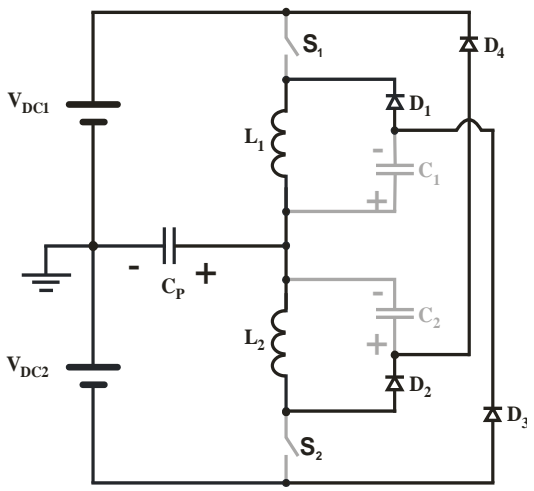

( b )

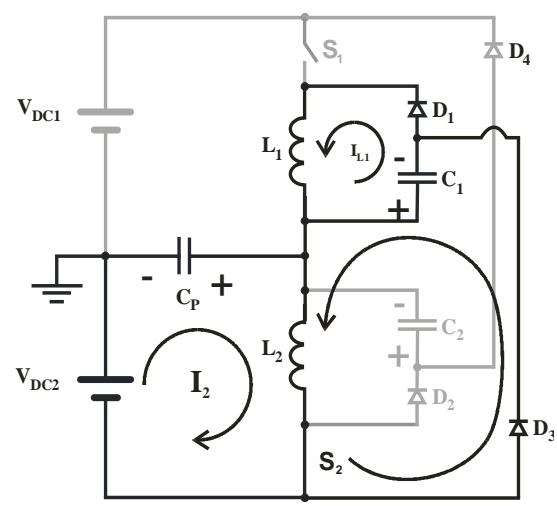

(c)

Figura 4: Etapas de Operação.

Este comportamento evita sobretensão nos interruptores $S_{1}$ e $S_{2}$, dispensa o uso de snubbers e, consequentemente, eleva o rendimento do amplificador.

Terceira Etapa: neste estágio a fonte de tensão $V_{D C 2}$, junto com a carga, ajuda a modular a forma de onda de tensão de saída como mostrado na Fig. 4 (c).

Seguindo estas etapas, a tensão de referência é modulada so- 


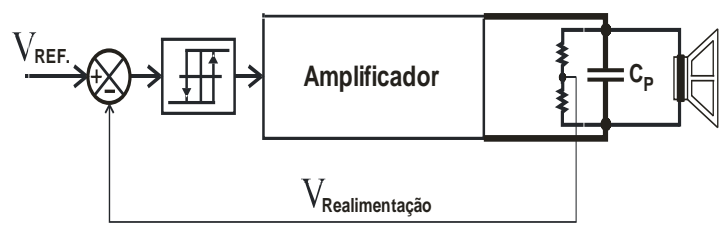

Figura 5: Diagrama de blocos do amplificador.

bre o capacitor $C_{P}$ e de acordo com as regras i e ii a tensão sobre os capacitores $C_{1}$ e $C_{2}$ é igual à tensão sobre o capacitor $C_{P}$ mais um nível cc fornecido pelas respectivas fontes de tensão $V_{D C 1}$ e $V_{D C 2}$.

Os sinais de gatilho dos interruptores $S_{1}$ e $S_{2}$ são obtidos por meio de laço de histerese entre a comparação da amostra de tensão do sinal de saída (tensão sobre o capacitor $C_{P}$ ) e a tensão de referência, como mostrado no diagrama de blocos da Fig. 5. Desta maneira, a forma de onda de tensão de saída segue o sinal de referência por meio de uma estratégia de controle de baixo custo.

Deste modo, a frequência de chaveamento, do conversor proposto, depende da dinâmica do circuito de realimentação e da rapidez do comparador utilizado. No protótipo implementado, a frequência de chaveamento está em torno de $50 \mathrm{kHz}$.

Um divisor resistivo é utilizado para medir a tensão de saída e o sinal é aplicado na entrada inversora de um comparador, implementado com um LM318, como mostrado na Fig. 6. O sinal de referência é aplicado na entrada não inversora do amplificador operacional e o resultado da comparação é usado para comandar os interruptores $S_{1}$ e $S_{2}$ de modo complementar.

\section{EQUAÇÕES PRINCIPAIS}

O valor máximo do "slew-rate" deve ser considerado de modo a se alcançar um projeto preciso do amplificador Classe D proposto. O "slew-rate" é a máxima taxa de variação $(d v / d t)$ da saída do amplificador por unidade de tempo. Este valor, na teoria, deveria ser infinito, o que na realidade não acontece. O "slew-rate" é o fator que limita a máxima frequência que pode ser amplificada, deste modo, o máximo "slew-rate" é determinado considerando a máxima frequência da forma de onda senoidal ou triangular a ser amplificada. É prática comum, em amplificadores de áudio, determinar o "slew-rate" máximo utilizando-se forma de onda senoidal, por ser facilmente gerada em frequências e amplitudes variáveis e por simplificar a elaboração de cálculos matemáticos.

Quanto maior a frequência da forma de onda a ser reproduzida, maior o "slew-rate" necessário, para que sejam reproduzidas, com baixa taxa de distorção harmônica. A forma de

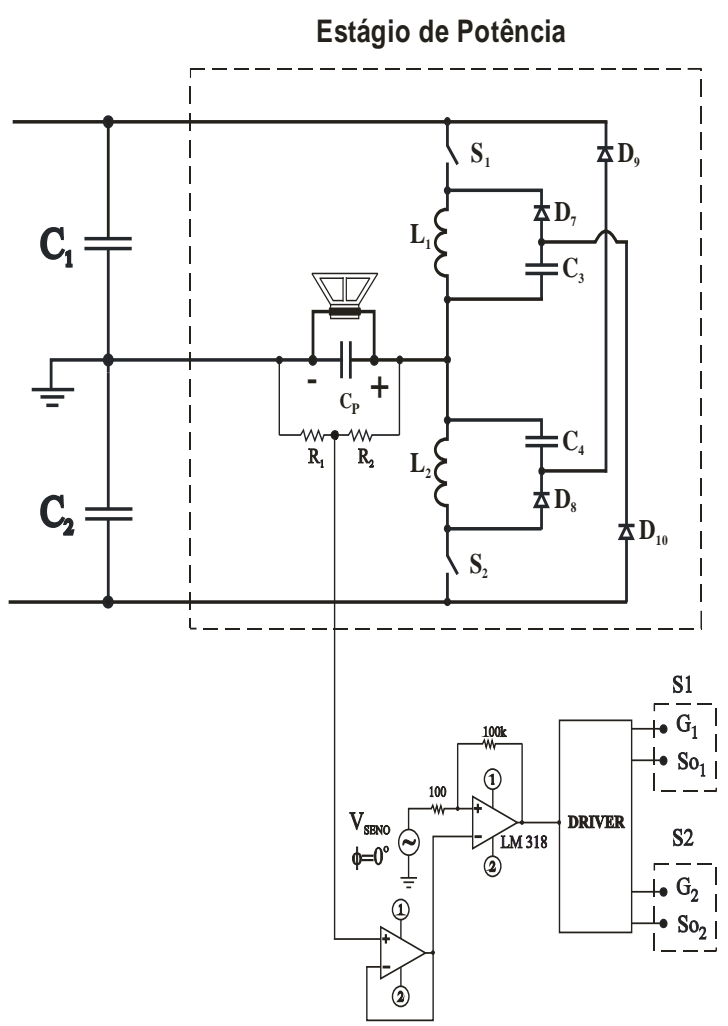

Figura 6: Diagrama esquemático do circuito de controle.

onda quadrada não pode ser considerada para o cálculo do máximo "slew-rate", pois teoricamente, esta forma de onda possui "slew-rate" infinito.

Por conseguinte, a equação do sinal instantâneo, a ser amplificado, é apresentada em (1).

$$
v_{C_{p}}(t)=V_{p k} \cdot \operatorname{sen}(\omega . t)
$$

Onde:

$v_{C_{p}}(t)$ - valor instantâneo de um sinal senoidal sobre o capacitor $C_{P}$;

$V_{p k}$ - valor de pico do sinal senoidal;

$\omega$ - frequência angular 2. $\pi . f$.

Portanto, o "slew-rate" desejado pode ser calculado por:

$$
\frac{d v(t)}{d t}=\omega \cdot V_{p k} \cdot \cos (\omega \cdot t)
$$

A máxima variação de tensão ocorre no cruzamento por zero para a máxima frequência a ser amplificada, de modo que 


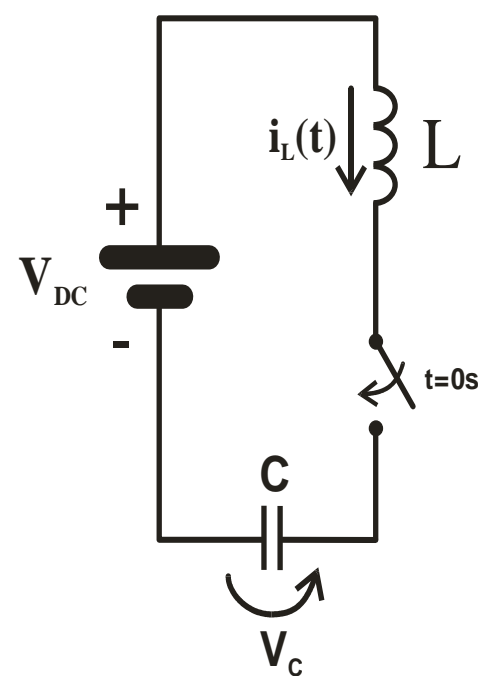

Figura 7: Circuito equivalente LC ("slew-rate" positivo).

$\cos (0)=1$. Isso significa que $\cos (\omega . t)$ presente na equação (2) pode ser substituido por 1 resultando em (3).

$$
\frac{d v(t)}{d t}=\omega \cdot V_{p k}
$$

A equação (4) relaciona corrente e capacitância e é importante para calcular a corrente necessária para produzir o "slew-rate" desejado no capacitor de filtro de saída.

$$
I_{p k}=C \cdot \frac{d v(t)}{d t}
$$

Combinando (3) e (4) resulta em:

$$
I_{p k}=2 . \pi \cdot f_{\max } \cdot C \cdot V_{p k}
$$

Onde:

$f_{\max }$ - frequência máxima do sinal amplificado pelo estágio modulador de largura de pulso.

O valor dos indutores $L_{1}$ e $L_{2}$, bem como o valor do capacitor $C_{P}, C_{1}$ e $C_{2}$ determinará o valor do "slew-rate" máximo do amplificador. Por este motivo, o circuito mostrado na Fig. 7 pode ser usado para encontrar a equação da corrente que produz o máximo "slew-rate".

Baseado no circuito LC série mostrado na Fig. 7 pode-se obter o valor de pico da corrente $i_{L(t)}$. A máxima corrente no indutor causa a máxima variação de tensão no capacitor $C$. A condição inicial foi indicada com o sub-índice 0 , por exemplo $V_{C 0}$ e $I_{C 0}$. Deste modo, a tensão sobre o indutor é:

$$
V_{L}(S)=S \cdot L \cdot I_{L(S)}-L \cdot I_{L_{0}}
$$

Sendo $V_{C 0}=q_{0} / C$ têm-se:

$$
V_{C}(s)=\frac{I_{L(S)}}{S \cdot C}+\frac{V_{C_{0}}}{S}
$$

Se $V_{D C}=V_{L}+V_{C}$, resulta em:

$$
\frac{V_{D C}}{S}=V_{L}(S)+V_{C}(S)
$$

Substituindo (6) e (7) em (8), obtém-se:

$$
I_{L}(S)=\frac{V_{D C}-V_{C 0}}{L\left(S^{2}+1 / L \cdot C\right)}+\frac{S \cdot I_{L 0}}{\left(S^{2}+1 / L \cdot C\right)}
$$

Como a frequência angular é:

$$
\omega_{0}=2 \cdot \pi \cdot f_{0}=\frac{1}{\sqrt{L \cdot C}}
$$

Então:

$$
I_{L}(S)=\frac{V_{D C}-V_{C 0}}{L \cdot \omega_{0}} \cdot \frac{\omega_{0}}{\left(S^{2}+\omega_{0}\right)}+I_{L 0} \frac{S}{\left(S^{2}+\omega_{0}\right)}
$$

Como a impedância característica do circuito ressonante é:

$$
Z_{0}=\sqrt{\frac{L}{C}}
$$

Então, aplicando-se a transformada inversa de Laplace à equação (11), a corrente instantânea sobre o indutor é:

$i_{L}(t)=I_{L 0} \cdot \cos \omega_{0}\left(t-t_{0}\right)+\frac{V_{D C}-V_{C 0}}{Z_{0}} \cdot \operatorname{sen} \omega_{0}\left(t-t_{0}\right)$

Para $I_{L 0}=V_{C 0}=0$, obtém-se:

$$
i_{L}(t)=\frac{V_{D C}}{\sqrt{L / C}} \cdot \operatorname{sen} \omega_{0}\left(t-t_{0}\right)
$$

A Equação 14 mostra que a corrente máxima no indutor ocorre quando $\operatorname{sen} \omega_{0}\left(t-t_{0}\right)=1$. Desta maneira, fazendo 


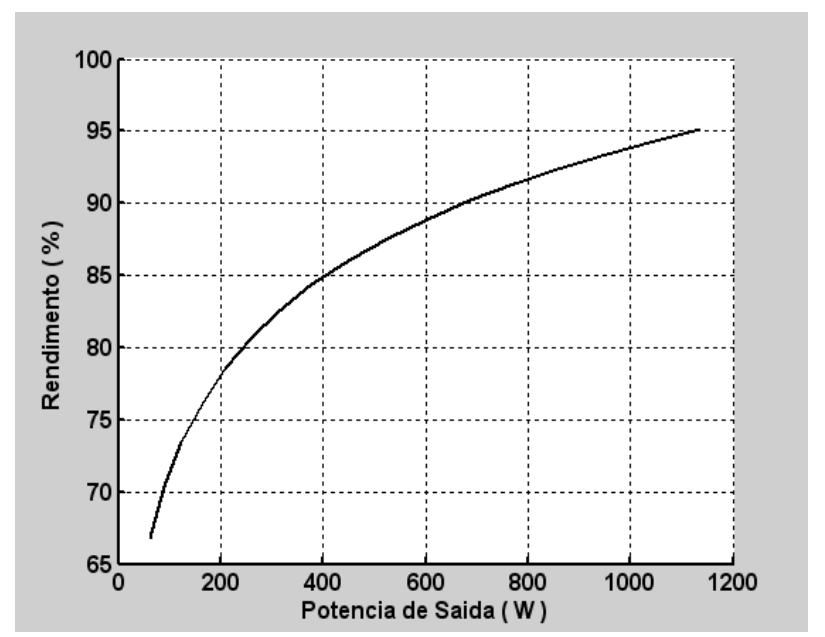

Figura 8: Curva do rendimento em função da potência de saída.

(5) igual a (14), obtém-se (15) que relaciona a indutância e a capacitância para se obter o "slew-rate" desejado.

$$
L \cdot C=\frac{V_{D C}^{2}}{4 \cdot \pi^{2} \cdot f_{\max }^{2} \cdot V_{p k}^{2}}
$$

É importante salientar que a impedância da carga não foi considerada porque a carga não é relacionada com o "slew-rate". Entretanto, a impedância da carga é relevante na análise da resposta em frequência e/ou do projeto do filtro.

A soma das capacitâncias $C_{P}, C_{1}$, e $C_{2}$ é igual à capacitância ressonante $C$. Valores apropriados para $C_{1}$ e $C_{2}$ são:

$$
C_{p} \leq\left(C_{1}=C_{2}\right) \leq 2 . C_{p}
$$

\section{RESULTADOS EXPERIMENTAIS}

A Fig. 8 mostra que o rendimento atinge $92 \%$ na condição de $800 \mathrm{~W}$ de potência de saída com carga de $8 \Omega$ e THD de $0,78 \%$, como pode ser observado na Fig. 9. A potência de $1.137 \mathrm{~W}$, exibida no gráfico das Fig. 8 e 9, foram extraídas com uma carga resistiva de $5 \Omega$ e frequência de 200 Hz. O teste de $1137 \mathrm{~W}$ foi possível porque tanto os indutores quanto os MOSFETs utilizados estavam dimensionados acima das especificações de projeto. Por fim, o diagrama de Bode da Fig. 10 mostra o ganho do amplificador em função da frequência. As frequências utilizadas nos experimentos para a elaboração do diagrama de Bode formam: 10, 100, 200, 400...20.000 Hz. A partir de $200 \mathrm{~Hz}$ a frequência sofreu acréscimo de $200 \mathrm{~Hz}$ até o valor final de $20 \mathrm{kHz}$.

A Fig. 11 apresenta a forma de onda de um sinal dente-de-

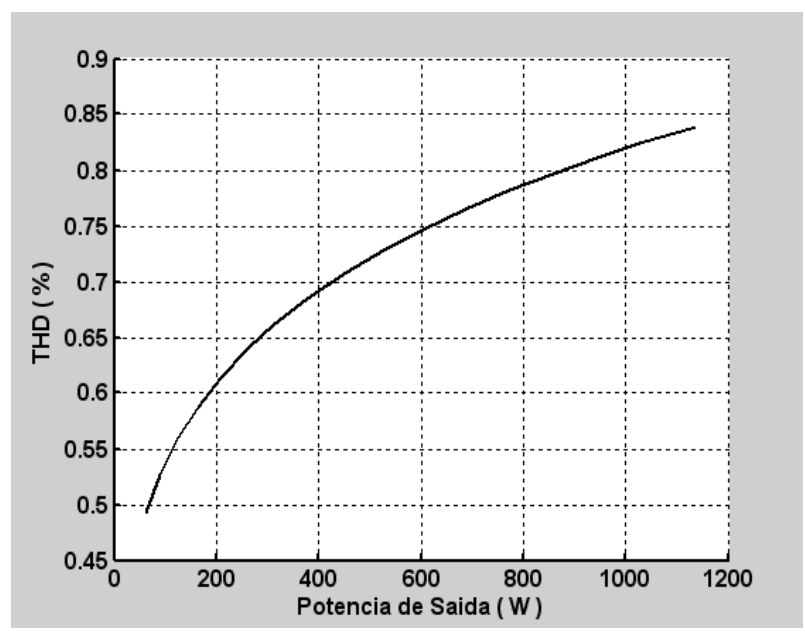

Figura 9: THD em função da potência de saída.

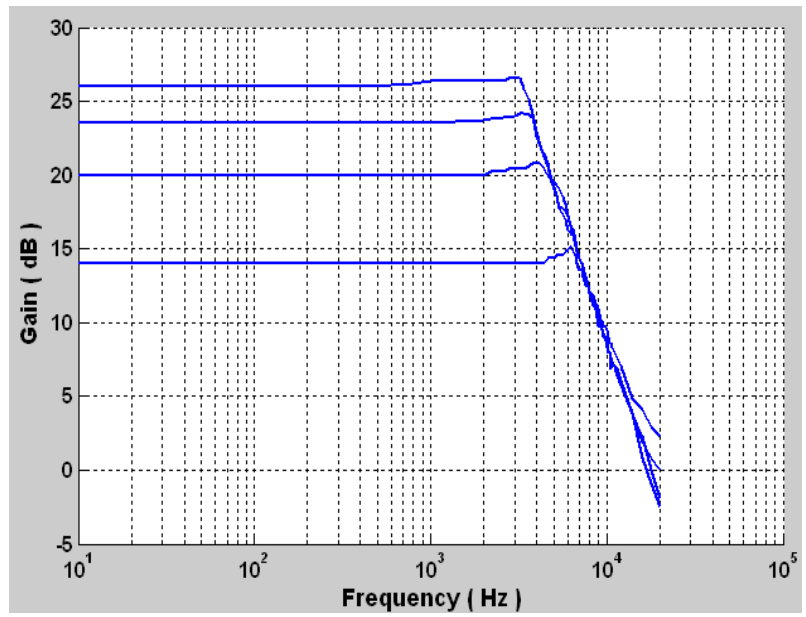

Figura 10: Curva do ganho em dB em função da frequência.

serra com $4 \mathrm{~V}$ de pico, aplicado na entrada do amplificador $\left(V_{R E F .}(t)\right)$ e o sinal amplificado correspondente com 100 $\mathrm{V}$ de pico $\left(V_{C P .}(t)\right)$. O experimento demonstra o comportamento do amplificador quando submetido a uma forma de onda com variação brusca da taxa de crescimento do sinal. A Fig. 12 apresenta o sinal amplificado e a forma de onda de corrente em uma carga resistiva de $20 \Omega$.

A Fig. 13 exibe o espectro de frequência, até a $13^{a}$ componente harmônica, do sinal dente-de-serra de referência e do sinal amplificado, onde observa-se a semelhança entre as amplitudes das componentes harmônicas dos sinais de entrada e de saída.

A Fig. 14 mostra o ensaio utilizado para medir o "slewrate" do amplificador. Usando uma onda quadrada como sinal, medimos a taxa de variação da rampa em seu ponto 


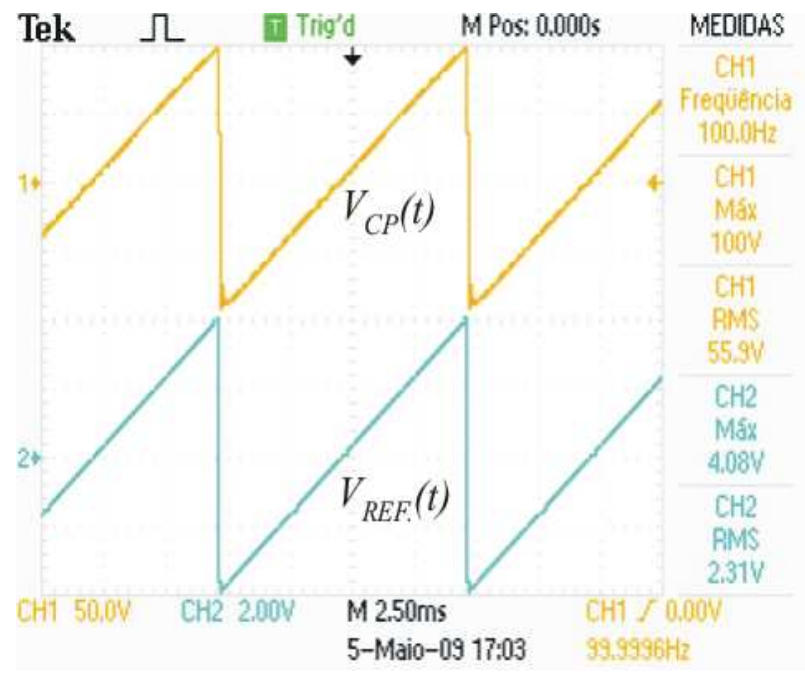

Figura 11: Sinal de referência - $V_{R E F}$. $(t)(2 \mathrm{~V} /$ div $)$ e sinal de referência amplificado $-V_{C P} .(t)(50 \mathrm{~V} /$ div $)$.

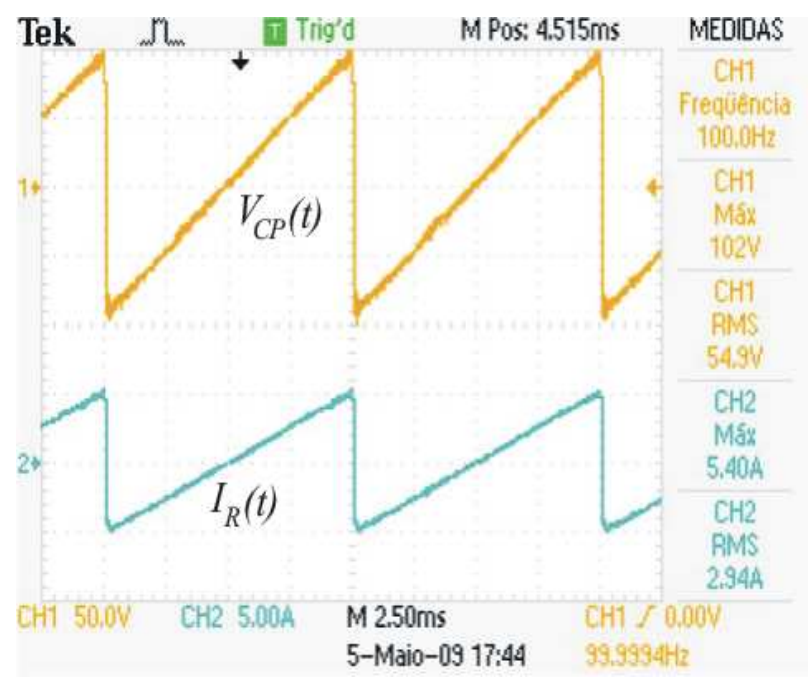

Figura 12: Sinal de referência amplificado - $V_{C P}(t)(50$ V/div) e corrente $-I_{R}(t)(5 \mathrm{~A} / \mathrm{div})$, em um resistor de 20 $\Omega$.

de máxima inclinação. O valor medido foi de $200 \mathrm{~V} \div$ $75 \mu \mathrm{s}=2,666 \mathrm{~V} / \mu \mathrm{s}$. O valor obtido é 5,33 vezes superior ao "slew-rate" do amplificador operacional de propósito geral LM741 que é de $0,5 \mathrm{~V} / \mu$ s, por exemplo.

\section{CONCLUSÃo}

Este artigo apresentou uma proposta de amplificador de áudio Classe D utilizado em subwoofer.

$\mathrm{O}$ amplificador proposto apresentou rendimento superior a $90 \%$, THD menor que $1 \%$ e não necessita de fonte de tensão

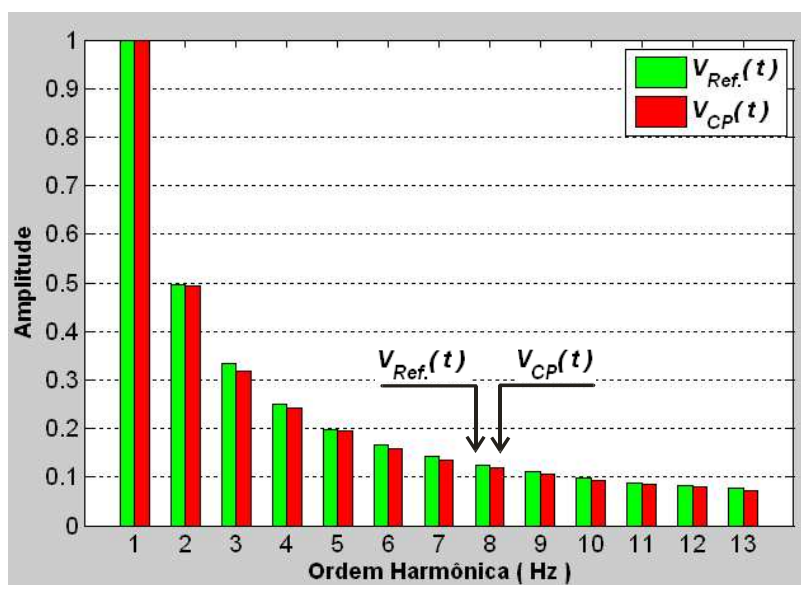

Figura 13: Espectro de frequência do sinal de referência $V_{R E F .}(t)$ e do sinal de referência amplificado - $V_{C P .}(t)$.

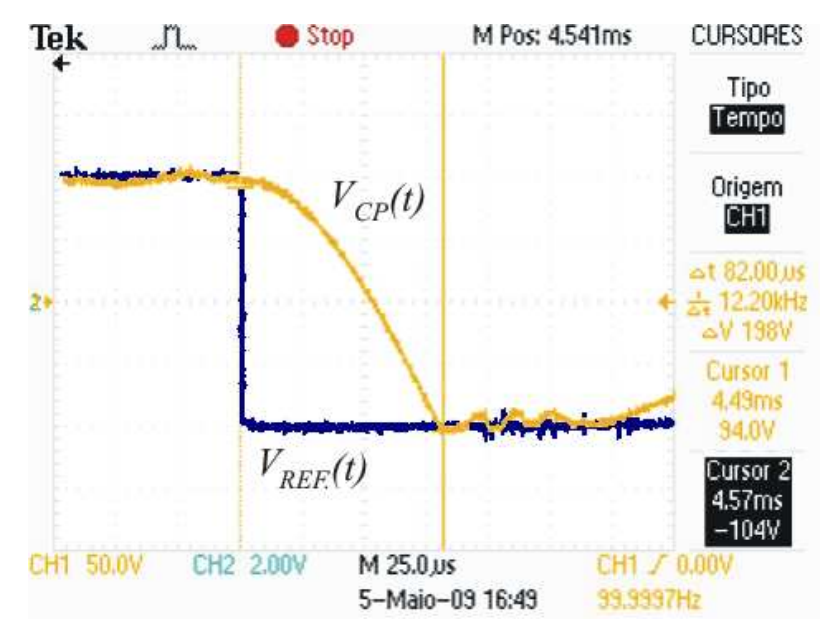

Figura 14: Medida de "slew-rate": Sinal de onda quadrada de referência - $V_{R E F \text {. }}(t)$ e detalhe da rampa - $V_{C P} .(t)$.

de alimentação regulada devido a realimentação implementada com um circuito de controle de baixo custo. Além disso, o amplificador proposto não necessita de filtro externo utilizado nos amplificadores de áudio convencionais para filtrar as componentes harmônicas dos pulsos PWM gerados pelo estágio de saída.

As principais vantagens da topologia proposta com relação a trabalhos anteriores são: circuito composto por apenas dois interruptores e, consequentemente, necessidade de somente dois circuitos de ataque de gatilho o que proporciona redução dos custos; não utiliza snubbers dissipativos; apresenta comutação não dissipativa.

Frequência de corte mais elevada, de modo a permitir a utilização do amplificador em woofers, pode ser obtida mudandose os valores de $\mathrm{L}$ e $\mathrm{C}$ do circuito de potência. 


\section{AGRADECIMENTOS}

Os autores gostariam de agradecer a CNPq, CAPES e FAPEMIG pelo suporte financeiro neste projeto e em muitos outros.

\section{REFERÊNCIAS}

Antunes, V. M. E., V. F. Pires and J. F. A Silva (2007). Narrow Pulse Elimination PWM for Multilevel Digital Audio Power Amplifiers Using Two Cascaded HBridges as a Nine-Level Converter, IEEE Transactions on Power Electronics, 22, (2), pp. 425-434.

Chang, J. S., M. T. Tan, Z. Cheng and Y. C. Tong (2000). Analisys and Design of Power Efficient Class D Amplifier Output Stages, IEEE Trans. on Circuits and Systems I: Fundamental Theory and Applications, 47(6), pp. 897-902.

Ertl, H., J. W. Kolar and F. C. Zach (1997). Basic consideration and topologies of switched-mode assisted linear power amplifiers, IEEE Trans. Ind. Electron., 44, pp. 116-123.

Gianart, A. E., R. M. Bass, W. Marshall Leach Jr and T. G. Habetl (2003). Analysis of the Class AD Audio Amplifier Including Hysteresis Effects, IEEE

Transactions on Power Electronics, 18, (2) pp. 679685.

Janssen, F. (2004). Excitação de Etapas de Potência Classe AB: Uma nova Abordagem, Eletrônica de Potência, 9(2), pp. 17-21.

Lai, Z. and K. M. Smedley (1996). A New Extension of OneCycle Control and Its Application to Switching Power Amplifiers, IEEE Transactions on Power Electronics, 11, (1), pp. 99-105.

Mioni, D. P., S. Finco e J. A. Pomílio (2007). Experimental results for a Low Voltage and High Efficiency Audio Power Amplifier Designed for Hearing Aids Applications, $9 \bigcirc$ Congresso Brasileiro de Eletrônica de Potência, pp. 978-983.

Nilsen, K. (1997). High-Fidelity PWM-Based Amplifier Concept for Active Loudspeaker Systems with Very Low Energy Consumption, Journal of Audio Engineering Society, 45, (7/8), pp. 554-570.

Oliva, A. R., S. A. Simon and V. Vo Thuy (2004). A Multi-Loop Voltage-Feedback Filterless Class-D Switching Audio Amplifier using Unipolar Pulse-WidthModulation, IEEE Transactions on Consumer Electronics, 50, (1), pp. 312-319.
Pascual, C., Z. Song, P. T. Krein, D. V. Sarwate, P. Midya and W. J. Roeckner (2003). High-Fidelity PWM Inverter for Digital Audio Amplification: Spectral Analysis, RealTime DSP Implementation, and Results, IEEE Transactions on Power Electronics, 18, (1), pp. 473-485..

Silva, F. V. R., J. B. Vieira Jr., E. A. A. Coelho and L. C. Freitas (1999a). A New Audio Switched Power Amplifier', Proceedings of IEEE INTELEC'1999 - Internation Telecommucations Energy Conference, Copenhege. Psicataway - NJ - USA, pp. 01-06.

Silva, F. V. R., J. B. Vieira Jr., E. A. A. Coelho and L. C. Freitas (1999b). A new switched Power Amplifier for High Power Applications, INTELEC - Internation Telecommucations Energy Conference Copenhage. Proceedings of IEEE'1999', Psicataway - NJ- USA: IEEE PRESS, pp. 01-06.

Walker, G. R. (2003). A Class B Switch-Mode Assisted Linear Amplifier, IEEE Transactions on Power Electronics, 18, (6), pp. 1278-1285. .

Yundt, G. B. (1986). Series or parallel-connected composite amplifiers, IEEE Trans. Power Electron., (PE-1), pp. $48-54$.

Zee, A. R. and E. Tuijl (1999). A power-efficiency audio amplifier combining switching and linear techniques, IEEE J. Solid-State Circuits, 34. 